\title{
New criteria for canonical number systems
}

\author{
by \\ Shigeki AkiYama (Niigata) and Hui Rao (Wuhan)
}

1. Introduction. Let $P(x)=p_{d} x^{d}+p_{d-1} x^{d-1}+\ldots+p_{0}$ be a polynomial of $x$ with integer coefficients and $p_{d}=1$. Let $R$ be the quotient ring $\mathbb{Z}[x] / P(x) \mathbb{Z}[x]$. As a $\mathbb{Z}$-module, $R$ is naturally isomorphic to $\mathbb{Z}^{d}$ and each element $\xi$ of $R$ is represented uniquely in the form

$$
\xi \equiv \sum_{i=0}^{d-1} a_{i} x^{i}(\bmod P(x))
$$

with $a_{i} \in \mathbb{Z}$. If an element $\xi \in R$ has an expression of the form

$$
\xi \equiv b_{0}+b_{1} x+\ldots+b_{M-1} x^{M-1}(\bmod P(x))
$$

with $a_{i} \in\left[0,\left|p_{0}\right|-1\right] \cap \mathbb{Z}$, then we say that $\xi$ has a canonical expression. If every element $\xi \in R$ has a canonical expression, then $P(x)$ is called a canonical number system generating polynomial, or a $C N S$ polynomial for short. Let $T: R \rightarrow R$ be a map defined by

$$
T(\xi) \equiv \sum_{i=0}^{d-1}\left(a_{i+1}-p_{i+1}\left[\frac{a_{0}}{p_{0}}\right]\right) x^{i}(\bmod P(x)) .
$$

Here we put $a_{d}=0$. Let $\alpha=x \bmod P(x)$. Then $R /(\alpha)$ is isomorphic to $\mathbb{Z} / p_{0} \mathbb{Z}$ by a $\mathbb{Z}$-module. Denote this isomorphism by $\tau: R /(\alpha) \rightarrow \mathbb{Z} / p_{0} \mathbb{Z}$. Then the map $T$ can be rewritten as

$$
T(\xi)=(\xi-a) / \alpha
$$

where $a \in\left[0,\left|p_{0}\right|-1\right]$ is the representative of $\tau(\xi)$. Denote by $T^{m}$ the $m$ th iteration of the map $T$. Then $\xi$ has a canonical expression (obviously this

2000 Mathematics Subject Classification: 11A63, 37B10.

Key words and phrases: canonical number system, radix representation, symbolic dynamics.

The first author is supported by the Japanese Ministry of Education, Culture, Sports, Science and Technology, Grand-in Aid for fundamental research, 14540015, 2002-2004.

The second author is supported by the Japan Society for the Promotion of Science. 
expression is unique) if and only if there is a non-negative integer $M$ such that $T^{M}(x)=0$.

When $P(x)$ is irreducible, $R$ is identified with $\mathbb{Z}[\alpha]$ with a root $\alpha$ of $P(x)$. This case has been extensively studied. In this case, $\left(\alpha,\left\{0,1, \ldots,\left|p_{0}\right|-1\right\}\right)$ is said to form a canonical number system if $P(x)$ is a CNS polynomial. Here we only refer to the original studies of I. Kátai \& J. Szabó [8], I. Kátai \& B. Kovács [6], [7], W. Gilbert [3] and B. Kovács \& A. Pethő [9].

A. Pethő [10] generalized this study to non-irreducible polynomials. The following is well known (see [9]):

If $P(x)$ is a CNS polynomial, then $P(x)$ is expanding (that is, each root of $P(x)$ has modulus greater than one) and $P(x)$ has no positive real root. In particular, the last condition implies

$$
p_{0}>1 \text {. }
$$

It is not hard to work out an algorithm determining whether a polynomial is a CNS polynomial. In Section 2, we will give such an algorithm. However, we want to see whether a given polynomial is a CNS polynomial by just looking its coefficients. Many papers are devoted to this problem. Generalizing former results of I. Kátai \& B. Kovács [6], [7], B. Kovács proved:

$$
\text { If } p_{0} \geq 2 \text { and }
$$

$$
p_{d} \leq p_{d-1} \leq \ldots \leq p_{0}
$$

then $P(x)$ is a CNS polynomial (see also [3], [10]) provided $P(x)$ is irreducible.

In S. Akiyama \& A. Pethő [1], it is proved that:

$$
p_{2} \geq 0, p_{3} \geq 0, \ldots, p_{d-1} \geq 0, \quad \sum_{i=1}^{d} p_{i} \geq 0, \quad \text { and } \quad p_{0}>2 \sum_{i=1}^{d}\left|p_{i}\right|
$$

imply that $P(x)$ is a CNS polynomial. In the same paper, they conjectured that the last condition can be relaxed to

$$
p_{0}>\sum_{i=1}^{d}\left|p_{i}\right|
$$

In this paper, we employ a method of Hollander to study CNS polynomials (he devised the method for the studying of the Pisot number system). In Section 3, we give two criteria for CNS polynomials. First we will give an affirmative answer to the conjecture of [1] and also deal with a slightly generalized situation

$$
p_{0} \geq \sum_{i=1}^{d}\left|p_{i}\right|
$$


in Theorem 3.2. Second, when $P(x)$ is a polynomial with (5) and has exactly one negative coefficient, $P(x)$ being a CNS polynomial is characterized by one inequality (see Theorem 3.5).

Section 4 is devoted to Brunotte's algorithm. H. Brunotte [2] discovered a nice method to determine CNS polynomials. The original argument looks not simple. Our method gives a short proof of Brunotte's Lemma (see Lemma 4.1). Also Theorem 4.2 shows that for any expanding $P(x)$, Brunotte's method actually gives a finite and efficient algorithm to determine CNS polynomials. Moreover, if the dominant condition (5) is assumed, then the algorithm becomes simpler (Theorem 4.3).

While preparing our paper, we were informed that similar results were shown recently via a different approach by K. Scheicher \& J. M. Thuswaldner [11]. We would like to express them our deep gratitude for the correspondence we had on this matter. It seems worthy to describe in detail the difference of ideas. The main difference is in the ways of description. Our way is algebraic having a flavor of symbolic dynamics. The idea of the proofs is originally due to M. Hollander [4]. On the other hand, their way depends on the transducer automata. Nevertheless, the basic ideas of the two papers are close to each other.

Corollary 4.4 is first proved in [11]. As a generalization of their result, we relax the condition (4) to (5) and get Theorem 4.3. Inspired by their result, easy characterizations of CNS polynomials with (4) of degree not larger than 5 will be given in Section 5 . Our idea in Section 5 is to use not only Corollary 4.4 but also all known necessary conditions to simplify our arguments. It is shown that the known necessary conditions are not sufficient to characterize degree five CNS polynomials, even if we assume (4).

2. Algorithm. Here we give a basic proposition.

Proposition 2.1. Assume that $P(x)$ is an expanding polynomial. Then for any $\xi \in R$, the sequence $\xi, T(\xi), T^{2}(\xi), \ldots$ is eventually periodic.

Remark 2.2. I. Kátai \& I. Környei [5] proved this in the case when $P(x)$ is expanding and irreducible.

Proof. Let $P(x)$ be an expanding polynomial as in Section 1 and $A$ be its companion matrix, that is,

$$
A=\left(\begin{array}{cccccc}
0 & 0 & 0 & \ldots & 0 & -p_{0} \\
1 & 0 & 0 & \ldots & 0 & -p_{1} \\
0 & 1 & 0 & \ldots & 0 & -p_{2} \\
\ldots \ldots & \ldots \ldots & \ldots \ldots & \ldots & \ldots . . \\
0 & \ldots & 0 & 1 & 0 & -p_{d-2} \\
0 & \ldots & \ldots & 0 & 1 & -p_{d-1}
\end{array}\right) .
$$


Let $D$ be a complete representative system of $\mathbb{Z}^{d} / A \mathbb{Z}^{d}$ of the form

$$
D=\left\{k \mathbf{v} \mid k=0,1, \ldots, p_{0}-1\right\}
$$

with $\mathbf{v}=(0, \ldots, 0,1)$. Let $\xi \in R$ and

$$
\xi=\xi_{0}+\xi_{1} \alpha+\ldots+\xi_{d-1} \alpha^{d-1} .
$$

We can embed $R$ into $\mathbb{R}^{d}$ by

$$
\pi(\xi):=\left(\begin{array}{c}
\xi_{0} \\
\xi_{1} \\
\vdots \\
\xi_{d-1}
\end{array}\right) .
$$

It is easy to check that $\pi(\alpha \xi)=A \pi(\xi)$. For any $y \in \mathbb{Z}^{d}$ there is a unique $v \in D$ such that $A^{-1}(y-v)$ is an integer. Define

$$
S(y):=A^{-1}(y-v) .
$$

To prove $\left\{T^{m}(\xi)\right\}_{m \geq 0}$ is eventually periodic, we only need to show that $\left\{S^{m}(y)\right\}_{m>0}$ is eventually periodic. As $A$ is expanding there exists a positive integer $k$ so that the map $f_{k}: x \mapsto A^{-k} x$ is a contraction $\left(^{1}\right)$ on $\mathbb{R}^{d}$. This implies that $\left\{S^{m}(y)\right\}_{m \geq 0}$ is a bounded sequence in $\mathbb{Z}^{d}$, and thus it is eventually periodic.

Let $\mathcal{P}$ be the set of purely periodic elements in $R$, i.e.,

$$
\mathcal{P}=\left\{\xi \in R \mid T^{M}(\xi)=\xi \text { for some } M>0\right\} .
$$

By Proposition 2.1, an expanding polynomial $P(x)$ is a CNS polynomial if and only if $\mathcal{P}=\{0\}$. It is important to get an algorithmic bound for searching elements of $\mathcal{P}$. In fact, it is easily seen that if $P(x)$ has no multiple root, then

$$
\mathcal{P} \subset\left\{\xi \in R|| \xi(\alpha) \mid \leq \frac{\left|p_{0}\right|-1}{|\alpha|-1} \text { for all roots } \alpha \text { of } P(x)\right\}
$$

(see [9], [5] and [10]). Here $\xi(\alpha)$ is well defined by substituting $\alpha$ for the indeterminate $x$.

In the following, we briefly discuss how to give an explicit upper bound suitable for computation, and this will give us an effective algorithm for determining whether a polynomial is a CNS polynomial.

Decompose the expanding polynomial $P(x)=\prod_{i=1}^{n}\left(x-\alpha_{i}\right)^{e_{i}+1}$ into factors in $\mathbb{C}(x)$. For $\xi \in R$, let

$$
T^{m}(\xi)=\frac{T^{m-1}(\xi)-a_{m-1}}{\alpha}
$$

$\left({ }^{1}\right)$ Note that $f_{1}$ is not necessarily a contraction. 
where $a_{m-1} \in\left[0,\left|p_{0}\right|-1\right] \cap \mathbb{Z}$ is a representative of $\tau\left(T^{m-1}(\xi)\right)$. Then

$$
\xi=a_{0}+a_{1} \alpha+\ldots+a_{m-1} \alpha^{m-1}+\alpha^{m} T^{m}(\xi) .
$$

We wish to give an upper bound of the set $\left\{T^{m}(\xi)\right\}_{m=0,1, \ldots}$. Put $\xi=$ $E(x) \bmod P(x)$ and $T^{m}(\xi)=F_{m}(x) \bmod P(x)$. Then $(8)$ is rewritten as

$$
E(x)=a_{0}+a_{1} x+\ldots+a_{m-1} x^{m-1}+x^{m} F_{m}(x)+G_{m}(x) P(x)
$$

for some $G_{m}(x) \in \mathbb{Z}[x]$. We claim that, for any $\varepsilon>0$,

$$
\left|\frac{d^{j}}{d x^{j}} F_{m}\left(\alpha_{i}\right)\right| \leq K_{j}\left(\alpha_{i}\right)+\varepsilon
$$

for a sufficiently large $m$, where

$$
K_{j}\left(\alpha_{i}\right)=\frac{j !\left(\left|p_{0}\right|-1\right)}{\left(\left|\alpha_{i}\right|-1\right)^{1+j}}
$$

This is shown by differentiating (9) several times and using the estimate

$$
\left|\sum_{l=1}^{m} a_{m-l} \frac{(-l)_{j}}{\alpha_{i}^{j+l}}\right| \leq\left(\left|p_{0}\right|-1\right) \sum_{l=1}^{m} \frac{l(l+1) \ldots(l+j-1)}{\left|\alpha_{i}\right|^{j+l}}=K_{j}\left(\alpha_{i}\right),
$$

where

$$
(r)_{j}= \begin{cases}r(r-1) \ldots(r-j+1), & j \geq 1 \\ 1, & j=0 .\end{cases}
$$

On the other hand, by (1), there exist integers $c_{m, i}$ such that

$$
F_{m}(x)=\sum_{i=0}^{d-1} c_{m, i} x^{i}
$$

Then we can deduce an upper bound of $c_{m, i}$ from (10). This shows that $\left\{T^{m}(\xi)\right\}_{m=0,1, \ldots}$ is contained in a bounded set, which gives an alternative proof of Proposition 2.1.

As $\xi=E(x) \bmod P(x)$ we may define, for $i=1, \ldots, e_{i}$,

$$
\xi^{(j)}\left(\alpha_{i}\right)=\left.\frac{d^{j}}{d x^{j}} E(x)\right|_{x=\alpha_{i}} .
$$

\section{Proposition 2.3.}

$$
\mathcal{P} \subset\left\{\xi \in R|| \xi^{(j)}\left(\alpha_{i}\right) \mid \leq K_{j}\left(\alpha_{i}\right) \text { for } i=1, \ldots, n, j=0,1, \ldots, e_{i}\right\} .
$$

Proof. If $\xi \in \mathcal{P}$, then there exists a positive integer $M$ such that $\xi=$ $T^{M}(\xi)$. Thus $\xi=T^{m}(\xi)=F_{m}(x) \bmod P(x) \in \mathcal{P}$ for any $m$ which is a multiple of $M$. This means that $\left|\xi^{(j)}\left(\alpha_{i}\right)\right| \leq K_{j}\left(\alpha_{i}\right)+\varepsilon$ for any $\varepsilon>0$, showing the assertion. 
3. Sufficient conditions on CNS. Put $\alpha=x \bmod P(x)$. Then by (1), $R$ has a base $\left\{1, \alpha, \alpha^{2}, \ldots, \alpha^{d-1}\right\}$ as a $\mathbb{Z}$-module. We introduce a different base $\left\{w_{1}, \ldots, w_{d}\right\}$, which already appeared in [2], [1], [11] and implicitly in $[3]$ :

$$
\left(\begin{array}{c}
w_{1} \\
w_{2} \\
w_{3} \\
\vdots \\
w_{d}
\end{array}\right)=\left(\begin{array}{cccccc}
p_{d} & 0 & \ldots & \ldots & \ldots & 0 \\
p_{d-1} & p_{d} & 0 & \ldots & \ldots & 0 \\
p_{d-2} & p_{d-1} & p_{d} & 0 & \ldots & 0 \\
\ldots \ldots \ldots \ldots \ldots \ldots \ldots \ldots \ldots \ldots \ldots . . . \\
p_{1} & p_{2} & p_{3} & \ldots & p_{d-1} & p_{d}
\end{array}\right)\left(\begin{array}{c}
1 \\
\alpha \\
\alpha^{2} \\
\vdots \\
\alpha^{d-1}
\end{array}\right) .
$$

Define $\iota: \mathbb{Z}^{d} \rightarrow R$ by $\iota\left(z_{1}, \ldots, z_{d}\right)=\sum_{i=1}^{d} z_{i} w_{i}$ and

$$
z_{d+1}=-\left[\frac{\sum_{i=1}^{d} z_{i} p_{d-i+1}}{p_{0}}\right] .
$$

Replace $\left(z_{1}, z_{2}, \ldots, z_{d}\right)$ by $\left(z_{2}, z_{3}, \ldots, z_{d+1}\right)$, where $z_{d+1}$ is determined by the above formula. In this way, once $\left(z_{1}, \ldots, z_{d}\right) \in \mathbb{Z}^{d}$ is given, it defines an infinite sequence $\left(z_{1}, z_{2}, \ldots, z_{d}, z_{d+1}, \ldots\right)$. Let $\sigma: \mathbb{Z}^{d} \rightarrow \mathbb{Z}^{d}$ be a "shift" map:

$$
\sigma\left(z_{1}, z_{2}, \ldots, z_{d}\right)=\left(z_{2}, z_{3}, \ldots, z_{d+1}\right) .
$$

Then we can easily obtain the commutavity of the following diagram:

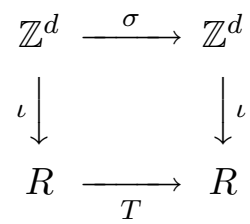

Hereafter we employ the method due to M. Hollander [4] developed for a different number system attached to Pisot numbers. His main idea is to interpret the map $T$ as a shift on bi-infinite words generated by $\mathbb{Z}$. The next proposition is merely a consequence of the definition of $z_{i}$ but we restate it to emphasize Hollander's idea.

Proposition 3.1. We have

$$
0 \leq z_{i} p_{d}+z_{i+1} p_{d-1}+\ldots+z_{i+d-1} p_{1}+z_{i+d} p_{0}<p_{0},
$$

and $z_{d+i}$ is uniquely determined by this condition.

Theorem 3.2. Assume that $P(x)$ is an expanding polynomial whose coefficients satisfy $p_{2} \geq 0, p_{3} \geq 0, \ldots, p_{d-1} \geq 0, \sum_{i=1}^{d} p_{i} \geq 0$ and the dominant condition $p_{0}>\sum_{i=1}^{d}\left|p_{i}\right|$. Then $P(x)$ is a CNS polynomial. The dominant condition can be replaced by $p_{0} \geq \sum_{i=1}^{d}\left|p_{i}\right|$ if one of the following conditions holds: 
- $p_{1}<0$,

- $p_{i}>0$ for all $i=1, \ldots, d-1$.

REMARK 3.3. The dominant condition $p_{0}>\sum_{i=1}^{d}\left|p_{i}\right|$ guarantees that the polynomial $P(x)$ is expanding (cf. [1, Lemma 1]).

REMARK 3.4. The above supplementary condition is necessary when $p_{0}=\sum_{i=1}^{d}\left|p_{i}\right|$. For example, $x^{3}+3 x^{2}+4$ is not a CNS polynomial. H. Brunotte kindly pointed out an error in the original manuscript and the example is also due to him.

The proof of Theorem 3.2 is divided into two parts. First we settle the case $p_{1} \geq 0$. The case $p_{1}<0$ will be shown in a more general form in Theorem 3.5.

Proof of Theorem 3.2 when $p_{1} \geq 0$. Recall that $\mathcal{P}$ is the set of purely periodic elements in $R$ defined by (6). To prove $P(x)$ is a CNS polynomial, it suffices to show that $\mathcal{P}=\{0\}$. Otherwise let $\xi=\sum_{i=0}^{d-1} z_{i} w_{i}$ be a non-zero element of $\mathcal{P}$ and $z_{0} z_{1} z_{2} \ldots$ be the infinite sequence determined by Proposition 3.1. Since $\xi$ is a non-zero purely periodic element, we have $z_{0} z_{1} z_{2} \ldots \neq 0^{\infty}$ and it is purely periodic. So we can extend it to be a bi-infinite word $\Xi=\ldots z_{-2} z_{-1} z_{0} z_{1} z_{2} \ldots$ and it is easy to see that

$$
0 \leq z_{i} p_{d}+z_{i+1} p_{d-1}+\ldots+z_{i+d-1} p_{1}+z_{i+d} p_{0}<p_{0}
$$

for all $i \in \mathbb{Z}$.

First we argue that there exist $i \in \mathbb{Z}$ such that $z_{i}<0$. For if $z_{i} \geq 0$ for all $i \in \mathbb{Z}$, then for an index $i$ such that $z_{i+d}>0$ we have

$$
z_{i} p_{d}+z_{i+1} p_{d-1}+\ldots+z_{i+d-1} p_{1}+z_{i+d} p_{0} \geq p_{0},
$$

which is a contradiction.

Let $\min _{i \in \mathbb{Z}} z_{i}=-\kappa \leq-1$ and $\max _{i \in \mathbb{Z}} z_{i}=\eta$. Note that both $\kappa$ and $\eta$ are finite since $\Xi$ is a periodic word. Now we take $i$ such that $z_{i+d}=-\kappa$. Then by the left inequality of (13),

$$
\begin{aligned}
z_{i} p_{d}+z_{i+1} p_{d-1}+\ldots+z_{i+d-1} p_{1} & \geq \kappa p_{0}, \\
\eta\left(p_{d}+p_{d-1}+\ldots+p_{1}\right) & \geq \kappa p_{0},
\end{aligned}
$$

which yields

$$
\eta>\kappa \geq 1
$$

provided $p_{0}>\sum_{i=1}^{d}\left|p_{i}\right|$.

We shall show that (16) holds in case $p_{i}>0$ for $i=1, \ldots, d-1$ and $p_{0}=\sum_{i=1}^{d} p_{i}$. The above argument shows

$$
\eta \geq \kappa \geq 1
$$


Assume $\eta=\kappa$. By (14) and (15), $\eta=\kappa$ and $p_{i}>0$ implies

$$
z_{i}=z_{i+1}=\ldots=z_{i+d-1}=\eta \text {. }
$$

Let us consider (13) with $i \rightarrow i-1$. By (17) and the right inequality of (13),

$$
\begin{gathered}
z_{i-1}+\eta\left(p_{d-1}+p_{d-2}+\ldots+p_{0}\right)<p_{0}, \\
-\kappa \leq z_{i-1}<-\eta\left(p_{d-1}+p_{d-2}+\ldots+p_{1}\right)+(1-\eta) p_{0}, \\
\kappa>\eta\left(p_{d-1}+p_{d-2}+\ldots+p_{1}\right) .
\end{gathered}
$$

As $p_{d-1}+p_{d-2}+\ldots+p_{1}>0$ we get $\kappa>\eta$, which is absurd. This shows $\eta>\kappa$ in any case.

Let $j$ be an index such that $z_{j+d}=\eta$. Now we use (13) again with $i=j$ to get

$$
\begin{aligned}
z_{j} p_{d}+z_{j+1} p_{d-1}+\ldots+z_{j+d-1} p_{1} & <(1-\eta) p_{0} \leq-\kappa p_{0}, \\
-\kappa\left(p_{d}+p_{d-1}+\ldots+p_{1}\right) & <-\kappa p_{0},
\end{aligned}
$$

which yields $p_{d}+p_{d-1}+\ldots+p_{1}>p_{0}$. This contradicts our assumption. Hence $\mathcal{P}=\{0\}$.

Inspecting the above proof, we get a necessary condition for $P(x)$ to be a CNS polynomial. Let $k$ be an integer, $0<k \leq d$, and consider the sum

$$
C_{k}(l)=\sum_{0 \leq k i+l \leq d} p_{k i+l}, \quad l=0,1, \ldots, k-1 .
$$

For a given $k$, if $C_{k}(l) \in\left[0, p_{0}-1\right]$ for all $l$, then $P(x)$ is not a CNS polynomial. Indeed, a bi-infinite word

$$
\Xi=(\overbrace{00 \ldots 0}^{k-1} 1)^{\infty}
$$

obviously gives an element of $\mathcal{P}$. Therefore, if $P(x)$ is a CNS polynomial then for any $k, 0<k \leq d$, there exist $l$ such that $C_{k}(l) \notin\left[0, p_{0}-1\right]$, which we call the $k$-subsum condition.

Since $P(x)$ has no positive roots, we see that $\sum_{i=0}^{d} p_{i} \geq 0$. Hence the 1 -subsum condition is nothing but

$$
\sum_{i=1}^{d} p_{i} \geq 0,
$$

which appeared in the condition of Theorem 3.2. This 1-subsum condition also appears in [1, Lemma 4].

Now let us treat polynomials having an isolated negative coefficient $p_{k}<0$ and satisfying the dominant condition $p_{0} \geq \sum_{i=1}^{d}\left|p_{i}\right|$. Under these assumptions, $C_{k}(l)(l>0)$ must be in $\left[0, p_{0}-1\right]$. Thus $C_{k}(0) \geq p_{0}$, i.e.,

$$
\sum_{1 \leq k i \leq d} p_{k i} \geq 0
$$


is necessary for $P(x)$ to be a CNS polynomial. (Note that this implies that $k$ is not greater than $d / 2$.) Theorem 3.5 shows that this condition is also sufficient.

TheOREM 3.5. Assume that $P(x)$ is an expanding polynomial with the dominant condition $p_{0} \geq \sum_{i=1}^{d}\left|p_{i}\right|$ whose coefficients are non-negative except $p_{k}<0$ for a single index $0<k<d$. Then $P(x)$ is a CNS polynomial if and only if

$$
\sum_{1 \leq k i \leq d} p_{k i} \geq 0
$$

As stated before, the proof of Theorem 3.2 for the case $p_{1}<0$ is completed at the same time.

Proof of Theorem 3.5. Suppose $P(x)$ is a polynomial satisfying the assumptions of the theorem and it is not a CNS polynomial. Then similarly to the proof of Theorem 3.2, we can construct a non-zero bi-infinite periodic word $\Xi=\ldots z_{-2} z_{-1} z_{0} z_{1} z_{2} \ldots$ satisfying (13). We shall derive a contradiction from the existence of such a word.

Let $\kappa=0$ if $z_{i} \geq 0$ for all $i \in \mathbb{Z}$. Otherwise we define $-\kappa=\min _{i \in \mathbb{Z}} z_{i}$. Let $\eta=\max _{i \in \mathbb{Z}} z_{i}$.

First we claim that $\eta>\kappa$. In case $\kappa=0$ this is trivial. Suppose $\kappa<0$. Let $i$ be an index such that $z_{i+d}=-\kappa$. Without loss of generality, we assume $i=0$. Then

$$
\begin{aligned}
0 & \leq z_{0} p_{d}+z_{1} p_{d-1}+\ldots+z_{d-1} p_{1}+z_{d} p_{0}<p_{0}, \\
\kappa p_{0} & \leq z_{0} p_{d}+z_{1} p_{d-1}+\ldots+z_{d-1} p_{1}, \\
\kappa p_{0} & \leq \kappa\left|p_{k}\right|+\eta \sum_{i \neq 0, k} p_{i} .
\end{aligned}
$$

Inequality (19) implies $\eta \geq \kappa$. Moreover if $z_{d-k} \neq-\kappa$, then we have the strict inequality

$$
\kappa p_{0}<\kappa\left|p_{k}\right|+\eta \sum_{i \neq k} p_{i}
$$

which implies $\eta>\kappa$. The remaining case is that $z_{d}=z_{d-k}=-\kappa$. If there is some $l>0$ such that $z_{d-k l} \neq-\kappa$, then by shifting indices, we may assume $z_{d}=-\kappa \neq z_{d-k}$, and $\eta>\kappa$ follows. If $z_{d-k l}=-\kappa$ for all $l=0,1,2, \ldots$, using the left inequality of (18), we have

$$
\kappa\left(p_{0}+p_{k}+\ldots+p_{k\left[\frac{d}{k}\right]}\right) \leq \eta \sum_{k \nmid i}\left|p_{i}\right|<\eta \sum_{i=1}^{d}\left|p_{i}\right| \leq \eta p_{0} .
$$

Hence $\kappa<\eta$ by the assumption $\sum_{1 \leq k i \leq d} p_{k i} \geq 0$. So our claim is proved.

Our next aim is to show that $z_{j}=\eta$ implies $z_{j+k l}=\eta$ for all $l \in \mathbb{Z}$. Without loss of generality, we may assume $z_{d}=\eta$. If $z_{d-k} \neq \eta$, then by the 
right inequality of (18),

$$
\begin{aligned}
p_{0} & >z_{0} p_{d}+z_{1} p_{d-1}+\ldots+z_{d-1} p_{1}+z_{d} p_{0} \\
& \geq-\kappa \sum_{\substack{i \neq 0, k \\
1 \leq i \leq d}}\left|p_{i}\right|-(\eta-1)\left|p_{k}\right|+\eta p_{0} \\
& \geq p_{0}+(\eta-1)\left(p_{0}-\sum_{1 \leq i \leq d} p_{i}\right) \geq p_{0} .
\end{aligned}
$$

This is a contradiction. So $z_{d+k l}=\eta$ for all $l \in \mathbb{Z}$. Now (18) becomes

$$
p_{0}>z_{0} p_{d}+z_{1} p_{d-1}+\ldots+z_{d-1} p_{1}+z_{d} p_{0} \geq-\kappa \sum_{k \nmid i}\left|p_{i}\right|+\eta \sum_{\substack{k \mid i \\ i \neq 0}} p_{k}+\eta p_{0} .
$$

By the assumption $\sum_{k \mid i, i \neq 0} p_{i} \geq 0$, we see $\kappa>0$. Moreover

$$
\kappa \sum_{k \nmid i}\left|p_{i}\right|>(\eta-1) p_{0} .
$$

This shows

$$
\sum_{1 \leq i \leq d}\left|p_{i}\right|>\sum_{\substack{1 \leq i \leq d \\ k \nmid i}}\left|p_{i}\right|>p_{0}
$$

which is a desired contradiction.

The classification of quadratic CNS polynomials $x^{2}+p_{1} x+p_{0}$ was already given in [6], [7], [3] and [12] in several ways. We reprove this result as an application of our discussion.

Corollary 3.6. Let $P(x)=x^{2}+p_{1} x+p_{0}$ be a quadratic polynomial. Then $P(x)$ is a CNS polynomial if and only if $-1 \leq p_{1} \leq p_{0}$ and $p_{0} \geq 2$.

Proof. If $P(x)$ is a CNS polynomial, then $p_{0} \geq 2$ by (2). Since there are no roots in $[-1,0]$, we have $P(-1)>0$, which shows $p_{1} \leq p_{0}$, and the 1-subsum condition implies $-1 \leq p_{1}$.

Conversely if $-1 \leq p_{1} \leq p_{0}$ and $p_{0} \geq 2$ then $P(x)$ must be expanding. If $p_{1}<p_{0}$, then Theorem 3.2 implies that $P(x)$ is a CNS polynomial as coefficients satisfy the dominant condition. The remaining case $p_{1}=p_{0}$ is settled by (3), a result of B. Kovács.

For an expanding polynomial $P(x)$, (7) gives an algorithmic bound of the set $\mathcal{P}$. If $P(x)$ satisfies the dominant condition, we can get a bound in another way. Theorem 3.7 is an improvement of Theorem 1 of S. Akiyama and A. Pethő [1], giving such a bound of $\mathcal{P}\left({ }^{2}\right)$.

$\left({ }^{2}\right)$ Though this is not explicitly mentioned, the bound of Theorem 1 of [1] is nothing but the bound for $\mathcal{P}$. This fact is easily seen from its proof. Note that we do not assume $p_{0}>0$. 
Theorem 3.7. Assume $\left|p_{0}\right|>\sum_{i=1}^{d}\left|p_{i}\right|$ and $\xi=\sum_{i=1}^{d} w_{i} z_{i} \in \mathcal{P}$. Then

$$
\left|z_{i}-\frac{p_{0}}{2 \sum_{i=0}^{d} p_{i}}\right| \leq \frac{\left|p_{0}\right|}{2\left(\left|p_{0}\right|-\sum_{i=1}^{d}\left|p_{i}\right|\right)} .
$$

Proof. First we prove the case $p_{0}>0$. Putting $\tau=p_{0} /\left(2\left(\sum_{i=0}^{d} p_{i}\right)\right)$, by (13), we have

$$
-p_{0} / 2 \leq \sum\left(z_{i+j}-\tau\right) p_{d-j}<p_{0} / 2 .
$$

Let $\eta=\max _{i \in \mathbb{Z}}\left|z_{i}-\tau\right|$ and choose $i$ such that $\eta=\left|z_{i+d}-\tau\right|$. If $z_{i+d}-\tau=-\eta$ then

$$
\eta\left(\left|p_{d}\right|+\left|p_{d-1}\right|+\ldots+\left|p_{1}\right|\right) \geq\left(\eta-\frac{1}{2}\right) p_{0} .
$$

If $z_{i+d}-\tau=\eta$ then

$$
-\eta\left(\left|p_{d}\right|+\left|p_{d-1}\right|+\ldots+\left|p_{1}\right|\right)<\left(\frac{1}{2}-\eta\right) p_{0} .
$$

Thus in any case, we have

$$
\eta \sum_{i=1}^{d}\left|p_{i}\right| \geq\left(\eta-\frac{1}{2}\right) p_{0}, \quad \eta \leq \frac{p_{0}}{2\left(p_{0}-\sum_{i=1}^{d}\left|p_{i}\right|\right)},
$$

which shows our assertion.

Now we wish to show the case $p_{0}<0$. For the moment, we permit a negative leading coefficient $p_{d}=-1$ and substitute $P(x)$ by $-P(x)$ to make $p_{0}>0$ and $p_{d}=-1$. Then we easily see that Proposition 3.1 remains true in the same notation. Thus the above proof also works for the case $p_{0}<0$.

4. Some remarks on H. Brunotte's result. H. Brunotte [2] found an interesting algorithm to determine whether a polynomial is a CNS polynomial. The original proof is not easy. Recently K. Scheicher and J. M. Thuswaldner [11] gave a simple proof of a similar result by using finite automata. In this section, we give another proof of Brunotte's Lemma based on the techniques of Section 3. The idea is inspired by [11]. Moreover, we give several remarks on Brunotte's algorithm.

Set

$$
\sigma^{*}\left(z_{1}, \ldots, z_{d}\right)=-\sigma\left(-z_{1},-z_{2}, \ldots,-z_{d}\right),
$$

where $\sigma$ is defined as in Section 3. Since $\sigma^{*}\left(z_{1}, \ldots, z_{d}\right)=\sigma\left(z_{1}+p_{0}-1\right.$, $\left.z_{2}, \ldots, z_{d}\right)$, Lemma 2 of $[2]$ reads

Lemma 4.1. Let $P(x)$ be a monic polynomial of degree $d$ with $p_{0} \geq 2$. If there is a set $E \subset \mathbb{Z}^{d}$ with the properties below, then $P(x)$ is a CNS polynomial $\left({ }^{3}\right)$ :

$\left({ }^{3}\right)$ There is a minor difference between (i) and the corresponding assumption of Lemma 2 in [2], which is $(0, \ldots, 0),(-1,0, \ldots, 0),(0, \ldots, 0,-1) \in E$. 
(i) $(0, \ldots, 0),(-1,0, \ldots, 0),(1,0, \ldots, 0) \in E$,

(ii) $\left(\sigma(E) \cup \sigma^{*}(E)\right) \subseteq E$,

(iii) for any $x \in E$, there exists a positive integer $M$ such that $\sigma^{M}(x)=0$.

Proof. Again let $\alpha=x \bmod P(x)$. Suppose $\xi \in R$ has a canonical expansion and $\eta \in \iota(E)$. We argue that $\xi+\eta$ also has a canonical expansion. Suppose

$$
T(\xi)=\frac{\xi-k_{1}}{\alpha}, \quad T(\eta)=\frac{\eta-k_{2}}{\alpha},
$$

where $k_{1}, k_{2} \in\left\{0,1, \ldots, p_{0}-1\right\}$. If $k_{1}+k_{2}<p_{0}$, then

$$
T(\xi+\eta)=\frac{\xi+\eta-\left(k_{1}+k_{2}\right)}{\alpha}=T(\xi)+T(\eta) .
$$

If $k_{1}+k_{2} \geq p_{0}$, then $k_{2}>0$ and

$$
T(-\eta)=\frac{-\eta-\left(p_{0}-k_{2}\right)}{\alpha} .
$$

So we have

$$
T(\xi+\eta)=\frac{\xi+\eta-\left(k_{1}+k_{2}\right)+p_{0}}{\alpha}=T(\xi)-T(-\eta) .
$$

By assumption (ii),

$$
-T(-\eta)=-\iota\left(\sigma\left(\iota^{-1}(-\eta)\right)\right)=\iota\left(\sigma^{*}\left(\iota^{-1}(\eta)\right)\right) \in \iota(E) .
$$

Repeating this argument, for any $n$ we have

$$
T^{n}(\xi+\eta)=T^{n}(\xi)+\eta^{*}
$$

for some $\eta^{*} \in \iota(E)$. Since $\xi$ has a canonical expansion, $T^{n}(\xi+\eta) \in \iota(E)$ for a large $n$. Now from assumption (iii), we conclude that $\xi+\eta$ has a canonical expansion.

As $\pm 1 \in \iota(E)$ by assumption (i), $\xi$ having a canonical expansion implies $\xi \pm 1$ have canonical expansions. Note that $\xi$ having a canonical expansion implies $\alpha \xi$ has a canonical expansion. Since every element of $\mathbb{Z}(x)$ can be obtained from 0 by these two operations, every element of $R$ has a canonical expansion.

This Lemma 4.1 gives a handy way to determine whether $P(x)$ is a CNS polynomial:

(a) Let $E_{1}=\{(0, \ldots, 0),(-1,0, \ldots, 0),(1,0, \ldots, 0)\}$.

(b) If $E_{i}$ is defined for $i<n$, then $E_{n}$ is defined by $E_{n}=E_{n-1} \cup$ $\sigma\left(E_{n-1}\right) \cup \sigma^{*}\left(E_{n-1}\right)$.

(c) If $E_{n} \neq E_{n-1}$ then continue with (b). If $E_{n}=E_{n-1}$ then proceed to (d).

(d) For each element $x$ of $E_{n}$, we confirm that there exists $M$ such that $T^{M}(x)=0$. 
Note that $E_{n}=-E_{n}$ for any $n$. When $P(x)$ is an expanding polynomial, the last process (d) will terminate in finitely many steps since the sequence $\left\{T^{i}(x)\right\}_{i=0,1,2, \ldots}$ is eventually periodic by Proposition 2.1.

Further it is important to point out that the above process (b) also terminates. Indeed, as $P(x)$ is expanding, we know that both $\sigma$ and $\sigma^{*}$ are eventually contractive. So the sets $E_{n}(n=1,2, \ldots)$ must be uniformly bounded. By the discreteness of $\mathbb{Z}^{d}$ in $\mathbb{R}^{d}$ and $E_{n} \supset E_{n-1}$ we conclude that the process (b) will certainly terminate. In particular, if $P(x)$ is separable then we can give a concrete bound of the sets $E_{n}$.

TheOREM 4.2. For $P(x)$ an expanding separable polynomial, let

$$
W=\left\{\xi \in R|| \xi(\theta) \mid \leq \frac{\left|p_{0}\right|-1}{|\theta|-1} \text { for all roots } \theta \text { of } P(x)\right\} .
$$

Then $E_{n} \subset \iota(W)$ for all $n$.

Proof. Let us go back to the representation in the base $\left\{1, \alpha, \ldots, \alpha^{d-1}\right\}$, consider the action of $T$ and define $T^{*}(\xi)=-T(-\xi)$. As $P(x)$ is expanding, $E_{1} \subset \iota(W)$ is clear. It suffices to show $T(W) \cup T^{*}(W) \subset W$. Indeed, this is equivalent to $\sigma(\iota(W)) \cup \sigma^{*}(\iota(W)) \subset \iota(W)$, and so we have $\sigma\left(E_{n}\right) \cup \sigma^{*}\left(E_{n}\right) \subset$ $\iota(W)$ provided $E_{n} \subset \iota(W)$.

One can easily see that $T(\xi)=\left(\xi-k_{1}\right) / \alpha$ and $T^{*}(\xi)=\left(\xi-k_{2}\right) / \alpha$ with $k_{1}, k_{2} \in\left[-p_{0}+1, p_{0}-1\right]$. Let $\theta$ be a root of $P(x)$. Then $T(\xi(\theta))=$ $\left(\xi(\theta)-k_{1}\right) / \theta$ and $T_{1}(\xi(\theta))=\left(\xi(\theta)-k_{2}\right) / \theta$. Put $K(\theta)=\left(\left|p_{0}\right|-1\right) /(|\theta|-1)$. Then $|\xi(\theta)| \leq K(\theta)$ implies

$$
\left|\frac{\xi(\theta)-k_{1}}{\theta}\right| \leq \frac{K(\theta)+p_{0}-1}{|\theta|}=K(\theta)
$$

showing $|T(\xi(\theta))| \leq K(\theta)$ and also $\left|T_{1}(\xi(\theta))\right| \leq K(\theta)$. This proves $T(W) \cup$ $T^{*}(W) \subset W$.

Thus we have another algorithm to determine whether a polynomial is CNS. The bound $W$ in Theorem 4.2 is the same as in (7). Thus this algorithm cannot be worse than the one in [9]. If we have a dominant condition as before, then we can say more.

THEOREM 4.3. Let $P(x)$ be a monic polynomial with dominant condition $p_{0} \geq \sum_{i=1}^{d}\left|p_{i}\right|$. Then $P(x)$ is a CNS polynomial if and only if every element of

$$
S=\left\{\xi \in R \mid \xi=\sum_{i=1}^{d} z_{i} w_{i} \text { and }\left|z_{i}\right| \leq 1\right\}
$$

has a canonical expression.

Proof. We need only show that the condition is sufficient. Let

$$
S^{\prime}=\left\{\left(z_{1}, \ldots, z_{d}\right) \mid z_{i} \in\{-1,0,1\}\right\} .
$$


Then $S^{\prime}$ has property (i) of Lemma 4.1. Under the dominant condition, it is easy to check that $S^{\prime}$ satisfies (ii). The assumption on $S$ implies that $S^{\prime}$ satisfies (iii). Hence $P(x)$ is a CNS polynomial.

Corollary 4.4. Let $P(x)$ be a monic polynomial satisfying $p_{0}>$ $\sum_{i=1}^{d}\left|p_{i}\right|$. Then $P(x)$ is a CNS polynomial if and only if every element of

$$
\left\{\xi \in R \mid \xi=\sum_{i=1}^{d} z_{i} w_{i} \text { and } z_{i}=0,1\right\}
$$

has a canonical expression.

Proof. Let $S^{\prime}$ be the set defined in Theorem 4.3. Pick any $\left(z_{1}, \ldots, z_{d}\right) \in$ $S^{\prime}$; it defines an infinite word $\left(z_{1}, \ldots, z_{d}, z_{d+1}, \ldots\right)$. The dominant condition $p_{0}>\sum_{i=1}^{d}\left|p_{i}\right|$ implies that $d_{i} \in\{0,1\}$ for any $i>d$. Hence there is an integer $M>0$ such that $\sigma^{M}\left(z_{1}, \ldots, z_{d}\right)=(0, \ldots, 0)$. Hence $P(x)$ is a CNS polynomial by Lemma 4.1.

\section{Characterizations of CNS polynomials with a dominant con-} dition. This section is inspired by the recent work by K. Scheicher \& J. M. Thuswaldner [11]. We shall give some simple necessary and sufficient conditions for CNS polynomials of degree 3,4 and 5 under the dominant condition $p_{0}>\sum_{i=1}^{d}\left|p_{i}\right|$.

Theorem 3 of S. Akiyama \& A. Pethő [1] says that

$$
p_{l}+\sum_{k=l+1}^{d}\left|p_{k}\right| \geq 0
$$

is a necessary condition for CNS polynomials with $p_{0} \geq \sum_{i=1}^{d}\left|p_{i}\right|$. The same idea allows us to show a slightly stronger assertion under the dominant condition (4). Namely, if $P(x)$ is a CNS polynomial then

$$
p_{l}+\sum_{\substack{k=l+1 \\ p_{k}>0}}^{d} p_{k} \geq 0
$$

under (4). To show it, the only thing to check is that the case $\varepsilon_{j}=-1$ does not occur under the notation of [1]. An analogous method allows us to show $\left({ }^{4}\right)$

$\left({ }^{4}\right)$ One might hope that $\sum_{k=l}^{d} p_{k} \geq 0$ for any $l=1, \ldots, d-1$ are necessary for a CNS polynomial $P(x)$. Unfortunately this is not the case. A counterexample is

$$
x^{10}-x^{9}+x^{8}-2 x^{7}+4 x^{6}+4 x^{5}+4 x^{4}-3 x^{3}-x^{2}+20,
$$

which is a CNS polynomial but $p_{d}+p_{d-1}+p_{d-2}+p_{d-3}<0$. 
Lemma 5.1. If $P(x)$ is a CNS polynomial satisfying $p_{0} \geq \sum_{i=1}^{d}\left|p_{i}\right|$, then $1+p_{d-1}+p_{d-2} \geq 0$.

Proof. If $d=2$, then $1+p_{d-1}+p_{d-2} \geq 0$ is clear. Thus we handle the case $d>2$. Assume that $1+p_{d-1}+p_{d-2}<0$ and $P(x)$ is a CNS polynomial. Since $1+p_{d-1} \geq 0$ by $(20)$, we have $p_{d-2}<0$. If $p_{d-1} \geq 0$ then $\left|p_{d}\right|+\left|p_{d-1}\right|+p_{d-2}<0$ gives a contradiction again by (20). Thus $p_{d-1}=-1$ and $p_{d-2}<0$. Put $T^{m}(x)=\sum_{i=0}^{d-1} T_{i}^{m}(x) \alpha^{j}$ for $x \in R$. Reviewing the definition of the basis $\left\{w_{i}\right\}$, we have $w_{j}=\sum_{k=0}^{j-1} p_{d+1+k-j} \alpha^{k}$. Using this we obtain $T_{i}^{m}(x)=\sum_{j=i}^{d-1} z_{j+m+1} p_{d+i-j}$ with $z_{j} \in \mathbb{Z}$ defined at the beginning of Section 3. Now we specify $x=-1$ and define an integer sequence $\left\{z_{i}\right\}_{i=1}^{\infty}$. By using (5), it is easily seen that $z_{i} \in\{0, \pm 1\}$. Our aim is to show that for any non-negative integer $m$, there exists $j$ that $T_{j}^{m}(-1)<0$, which proves that $P(x)$ is not a CNS polynomial. This is obviously true when $m=0$. If $T_{0}^{m-1}(-1) \geq 0$ then it is shown, as in Theorem 3 of [1], that there exists $j$ that $T_{j}^{m}(-1)<0$. Assume that $T_{0}^{m-1}(-1)<0$. By $(5)$, we have $z_{m+d}=1$. If $z_{m+d-1} \leq 0$, then

$$
T_{d-2}^{m}(-1)=z_{m+d-1}-z_{m+d}<0 .
$$

If $z_{m+d-1}>0$, then

$$
T_{d-3}^{m}(-1)=z_{m+d-2}-z_{m+d-1}+z_{m+d} p_{d-2} \leq 1-1+p_{d-2}<0 .
$$

Thus we have shown the lemma.

Here it may be convenient to summarize necessary conditions for CNS polynomials with (5).

TheOREM 5.2. Let $P(x)$ be an expanding polynomial with the dominant condition (5). Then $P(x)$ being a CNS implies

(a) $1+p_{d-1} \geq 0$

(b) $1+p_{d-1}+p_{d-2} \geq 0$;

(c) $\sum_{i=1}^{d} p_{i} \geq 0$;

(d) $\sum_{2 \mid i, 1 \leq i \leq d} p_{i} \geq 0$.

Proof. Lemma 5.1 and (20) imply (a) and (b). Condition (c) follows from the 1-subsum condition. We need only prove (d). Suppose not; then by (c), we know that $\sum_{2 \nmid i, 1 \leq i \leq d} p_{i} \geq 0$. By $(5), P(x)$ is not a CNS polynomial since it does not satisfy the 2 -subsum condition.

Theorem 5.3. Let $P(x)=x^{3}+p_{2} x^{2}+p_{1} x+p_{0} \in \mathbb{Z}[x]$ with $p_{0}>$ $1+\left|p_{2}\right|+\left|p_{1}\right|$. Then $P(x)$ is a CNS polynomial if and only if $p_{2} \geq 0$ and $1+p_{2}+p_{1} \geq 0$.

Proof. Assume that $P(x)$ is a CNS polynomial. Then Theorem 5.2(b) or (c) implies $1+p_{2}+p_{1} \geq 0$ and (d) gives $p_{2} \geq 0$. (These facts were shown in a 
different way in Proposition 1 of [1].) The sufficiency follows from Theorem 3.2 .

TheOREM 5.4. Let $P(x)=x^{4}+p_{3} x^{3}+p_{2} x^{2}+p_{1} x+p_{0}$ be a polynomial in $\mathbb{Z}[x]$ with $p_{0}>1+\left|p_{3}\right|+\left|p_{2}\right|+\left|p_{1}\right|$. Then $P(x)$ is a CNS polynomial if and only if the following five conditions hold:

$$
\begin{aligned}
p_{3} & \geq-1, \\
p_{2} & \geq-1, \\
p_{3}+p_{2} & \geq-1, \\
1+p_{3}+p_{2}+p_{1} & \geq 0, \\
p_{3}=-1 & \Rightarrow p_{1} \leq-2 .
\end{aligned}
$$

Proof. Assume that $P(x)$ is a CNS polynomial. Theorem 5.2 says the first four conditions are necessary. Let $p_{3}=-1$. Then $1+p_{3}+p_{2} \geq 0$ implies $p_{2} \geq 0$. Let us consider the 3 -subsum condition. As $p_{3}+p_{0}$ and $p_{2}$ are in $\left[0, p_{0}-1\right] \cap \mathbb{Z}$, we see that $1+p_{1}$ must be negative.

Now we show the sufficiency. Note that $1+p_{3}+p_{2} \geq 0$ implies that $p_{2}$ and $p_{3}$ are not both negative. First we consider the case $p_{3}=-1$. Then $p_{1} \leq-2$ and $p_{2} \geq 2$. The proof is done by drawing a directed graph consisting of $2^{4}=16$ vertices formed by $\left(z_{i}, z_{i+1}, z_{i+2}, z_{i+3}\right)$ with $z_{i} \in\{0,1\}$. Each vertex $\left(z_{i}, z_{i+1}, z_{i+2}, z_{i+3}\right)$ represents an element of

$$
\left\{\xi \in R \mid \xi=\sum_{i=1}^{4} z_{i} w_{i} \text { and } z_{i}=0,1\right\},
$$

which forms a test set in Corollary 4.4. We draw an edge from $\left(z_{i}, z_{i+1}, z_{i+2}\right.$, $\left.z_{i+3}\right)$ to $\left(z_{i+1}, z_{i+2}, z_{i+3}, z_{i+4}\right)$ if there is a possibility that

$$
\sigma\left(z_{i}, z_{i+1}, z_{i+2}, z_{i+3}\right)=\left(z_{i+1}, z_{i+2}, z_{i+3}, z_{i+4}\right)
$$

under these five conditions. This is depicted in Figure 1. Here we omit the loop from $(0, \ldots, 0)$ to itself. As this graph is a directed tree with a single terminal vertex $(0,0,0,0)$, we have completed the case $p_{3}=-1$. Second we treat the case $p_{3} \geq 0$ and $p_{2}=-1$. If $p_{1} \geq 0$, then as $1+p_{2} \geq 0$ we can apply Theorem 3.5 to show that $P(x)$ is a CNS polynomial. Let $p_{1} \leq-1$. As $1+p_{3}+p_{2}+p_{1} \geq 0$, we have $p_{3} \geq 1$ and $p_{3}+p_{1} \geq 0$. Figure 2 gives a similar directed graph showing that $P(x)$ is a CNS polynomial.

Finally if $p_{3} \geq 0$ and $p_{2} \geq 0$ then as $1+p_{3}+p_{2}+p_{1} \geq 0$ we can apply Theorem 3.2 to see that $P(x)$ is a CNS polynomial.

REMARK 5.5. The proofs of Theorems 5.3 and 5.4 show that a CNS polynomial with the dominant condition (4) is completely characterized by the known necessary conditions: $k$-subsum condition, (20) and Lemma 5.1 provided the degree of the polynomial is less than 5 . 


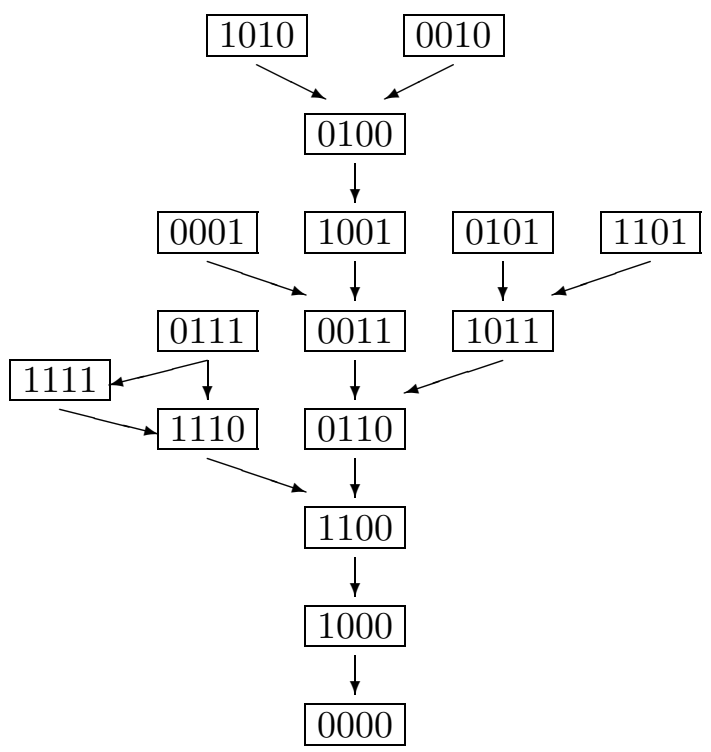

Fig. 1. $p_{3}=-1$, quartic case

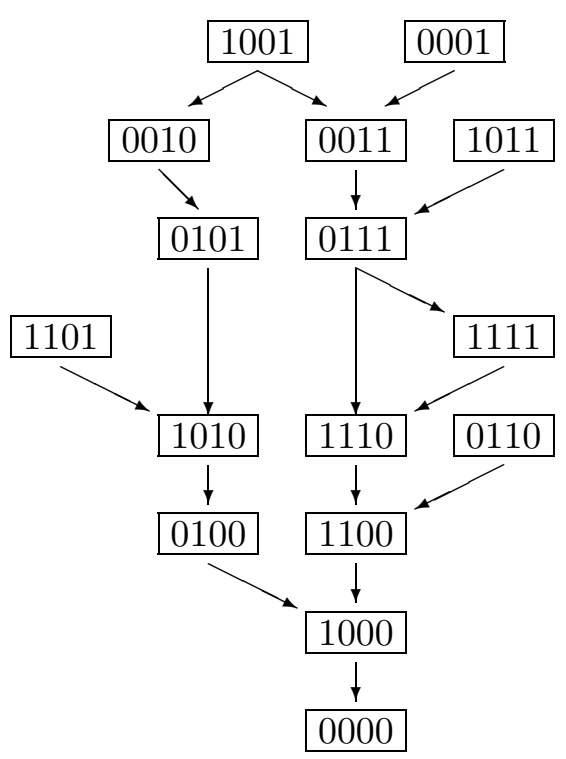

Fig. 2. $p_{2}=-1, p_{1}<0$, quartic case

TheOREM 5.6. Let $P(x)=x^{5}+p_{4} x^{4}+p_{3} x^{3}+p_{2} x^{2}+p_{1} x+p_{0}$ be a polynomial in $\mathbb{Z}[x]$ with $p_{0}>1+\left|p_{4}\right|+\left|p_{3}\right|+\left|p_{2}\right|+\left|p_{1}\right|$. Then $P(x)$ is a CNS polynomial if and only if the following five conditions hold: 


$$
\begin{aligned}
p_{2}+p_{4} & \geq 0, \\
1+p_{4}+p_{3}+p_{2}+p_{1} & \geq 0, \\
p_{4}<0 & \Rightarrow p_{4}=-1, p_{3} \geq 1, p_{1} \leq-2, \\
p_{3}<0, p_{1}+p_{4} \geq 0 & \Rightarrow p_{3} \geq-1, p_{2} \leq-2, \\
p_{3}<0, p_{1}+p_{4}<0 & \Rightarrow p_{4} \geq 0, p_{4}+p_{3} \geq 0 .
\end{aligned}
$$

Proof. Assume that $P(x)$ is a CNS polynomial. The first two conditions are shown in Theorem 5.2. Assume that $p_{4}<0$. In this case we see by $1+p_{4} \geq 0$ and $1+p_{4}+p_{3} \geq 0$ that $p_{4}=-1$ and $p_{3} \geq 0$. Now, $p_{2}+p_{4} \geq 0$ shows $p_{2} \geq 1$. By the 4 -subsum condition, $1+p_{1}$ must be negative. Further we can show that $p_{3} \geq 1$. For if $p_{3}=0$ then the 1 -subsum condition implies $p_{2}+p_{1} \geq 0$ and so

$$
\Xi=(0011)^{\infty}
$$

gives an element of $\mathcal{P}$. Thus we have shown the third necessary condition of Theorem 5.6.

Next consider the case $p_{3}<0$. As stated above, we have $p_{4} \geq 0$. Further assume that $p_{1}+p_{4} \geq 0$. By the 3 -subsum condition, $1+p_{2}<0$. Also $p_{3} \geq-1$. For if $p_{3} \leq-2$ then we can construct a bi-infinite word

$$
\Xi= \begin{cases}(01001)^{\infty}, & p_{3}+p_{1} \geq 0, \\ (01001011)^{\infty}, & p_{3}+p_{1}<0,\end{cases}
$$

which corresponds to an element of $\mathcal{P}$. This shows the 4 th necessary condition. Assume that $p_{3}<0$ and $p_{1}+p_{4}<0$. If $p_{4}+p_{3}<0$ then we see that

$$
\Xi= \begin{cases}(1001100)^{\infty}, & 1+p_{4}+p_{1} \geq 0 \\ (1001)^{\infty}, & 1+p_{4}+p_{1}<0\end{cases}
$$

is a corresponding word of a non-zero element of $\mathcal{P}$. Thus we have proved the five necessary conditions.

We now prove the sufficiency. First note that $p_{4}$ or $p_{3}$ cannot be an isolated negative coefficient by the claim before Theorem 3.5. Since $1+p_{4}+$ $p_{3}+p_{2}+p_{1} \geq 0$ and $p_{2}+p_{4} \geq 0$ are already assumed, Theorems 3.2 and 3.5 can be applied when there is at most one negative coefficient. Thus we only need to show the sufficiency in the case that there are at least two negative coefficients. In such 4 subcases, we can write down similar directed graphs used in the proof of Theorem 5.4:

(i) $p_{4}=-1$,

(ii) $p_{3}<0$ and $p_{1}+p_{4} \geq 0$,

(iii) $p_{3}<0$ and $p_{1}+p_{4}<0$,

(iv) $p_{4} \geq 0, p_{3} \geq 0, p_{1}<0$ and $p_{2}<0$.

In fact, case (i) is settled in Figure 3. In case (ii), it is easy to obtain the directed graph of Figure 4. Here we performed out-going amalgamation for 


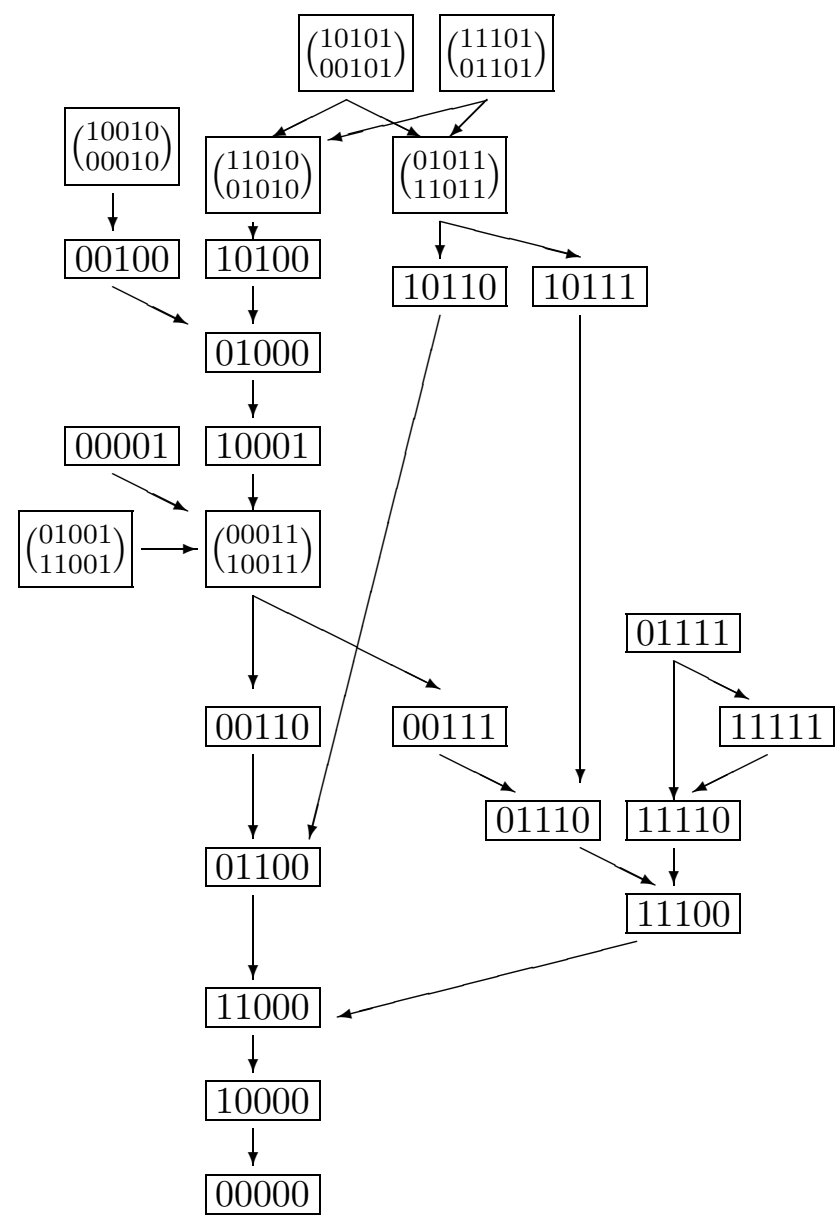

Fig. 3. $p_{4}=-1$, degree 5

some vertices on the original graphs to simplify them. This means that if two vertices $v_{1}, v_{2}$ have exactly the same successor vertices then such vertices are unified into one vertex $\left(v_{1}, v_{2}\right)$ in their graph expression. The last two cases are also dealt with easily. We leave these cases to the reader.

REMARK 5.7. Theorem 5.6 is restated as follows under the same condition. The polynomial $P(x)$ is a CNS polynomial if and only if it satisfies the $k$-subsum condition (for $k=1,2,3,4$ ), $p_{4} \geq-1, p_{3}+p_{4} \geq 0$ and

$$
p_{1}+p_{4} \geq 0 \Rightarrow p_{3} \geq-1 \text {. }
$$

To show this, we need only review the above proof that all five conditions in Theorem 5.6 can be derived from these conditions.

Note that the last two conditions cannot be obtained by combining $k$ subsum conditions, (20) and Lemma 5.1. Thus unfortunately, the known 


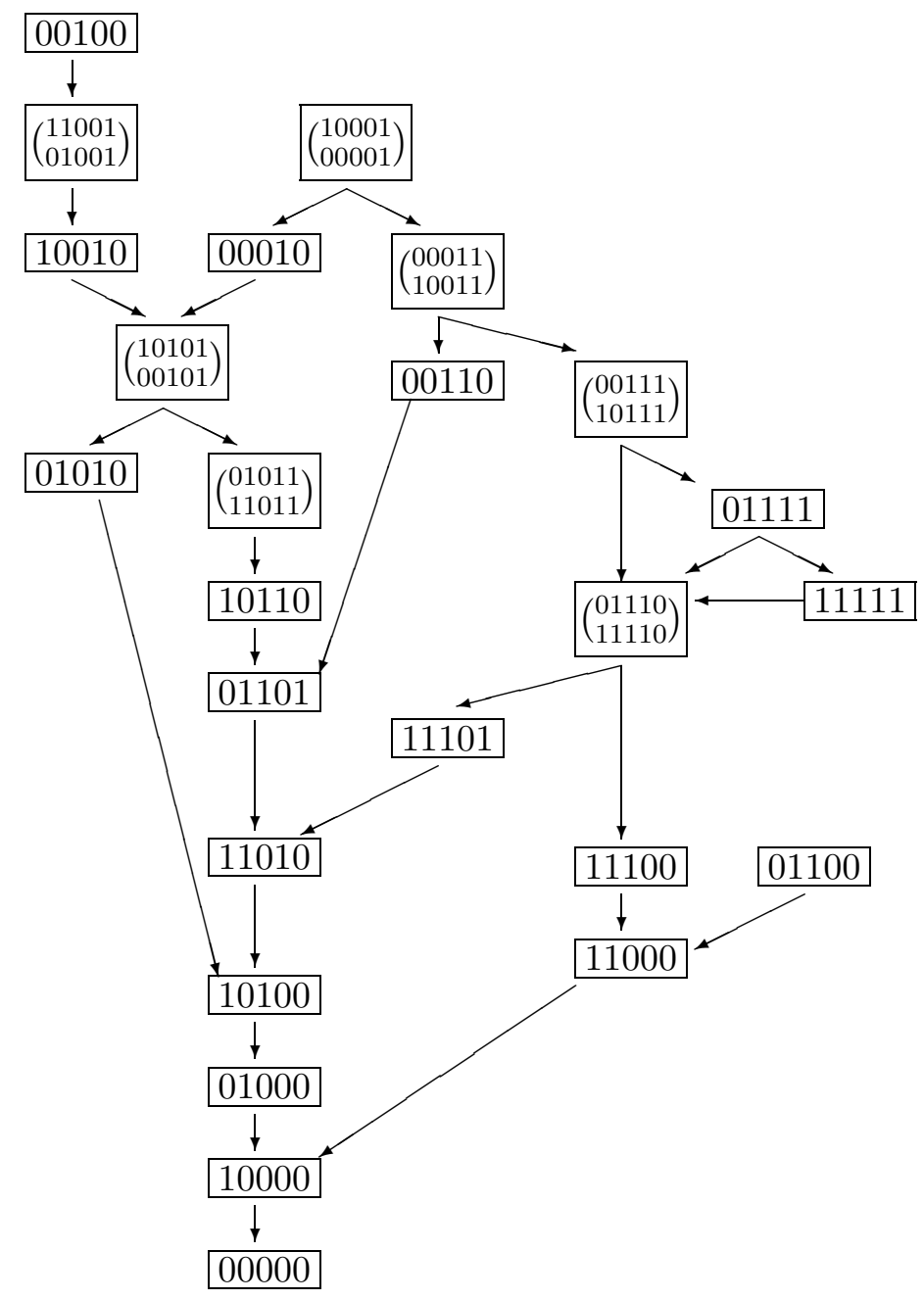

Fig. 4. $p_{4} \geq 0, p_{3}<0, p_{1}+p_{4} \geq 0$, degree 5

necessary conditions on CNS are not enough to characterize CNS polynomials of degree 5 .

REMARK 5.8. Fix a positive integer $d$ and consider expanding polynomials of degree $d$ under the dominant condition (4). We may say that Corollary 4.4 provides an algorithm to describe all such CNS polynomials. However it seems impractical for large degrees.

Indeed, possible lengths of periods of $\mathcal{P}$ are not larger than $2^{d}$. So we can characterize polynomials which admit non-zero periodic words $\Xi$ by finite sets of inequalities. Thus solving all these inequalities, we can describe the set of all CNS polynomials by ruling out such a family of non-CNS polynomials. 


\section{References}

[1] S. Akiyama and A. Pethő, On canonical number systems, Theoret. Comput. Sci. 270 (2002), 921-933.

[2] H. Brunotte, On trinomial basis of radix representations of algebraic integers, Acta Sci. Math. (Szeged) 67 (2001), 407-413.

[3] W. J. Gilbert, Radix representations of quadratic number fields, J. Math. Anal. Appl. 83 (1981), 263-274.

[4] M. Hollander, Linear numeration systems, finite beta expansions, and discrete spectrum of substitution dynamical systems, doctoral thesis, Washington Univ.

[5] I. Kátai and I. Környei, On number systems in algebraic number fields, Publ. Math. Debrecen 41 (1992), 289-294.

[6] I. Kátai and B. Kovács, Canonical number systems in imaginary quadratic fields, Acta Math. Hungar. 37 (1981), 159-164.

[7] —, - Kanonische Zahlensysteme in der Theorie der quadratischen Zahlen, Acta Sci. Math. (Szeged) 42 (1980), 99-107.

[8] I. Kátai and J. Szabó, Canonical number systems for complex integers, ibid. 37 (1975), 255-260.

[9] B. Kovács and A. Pethő, Number systems in integral domains, especially in orders of algebraic number fields, ibid. 55 (1991), 287-299.

[10] A. Pethő, On a polynomial transformation and its application to the construction of a public key cryptosystem, in: Computational Number Theory, A. Pethő et al. (eds.), de Gruyter, 1991, 31-44.

[11] K. Scheicher and J. M. Thuswaldner, On the characterization of canonical number system, Osaka J. Math., to appear.

[12] J. M. Thuswaldner, Elementary properties of canonical number systems in quadratic fields, in: Applications of Fibonacci Numbers, Vol. 7 (Graz, 1996), Kluwer, Dordrecht, 1998, 405-414.

Department of Mathematics

Faculty of Science

Niigata University

Ikarashi 2-8050

Niigata 950-2181, Japan

E-mail: akiyama@math.sc.niigata-u.ac.jp
Department of Mathematics Faculty of Science Wuhan University 430072, Wuhan, P.R. China E-mail: myhacone@yahoo.com

Received on 27.11.2001

and in revised form on 21.1.2003 